\title{
On second-order differential subordinations for a class of analytic functions defined by convolution
}

\section{Arzu Akgül}

Kocaeli University, Faculty of Arts and Sciences, Department of Mathematics, Umuttepe Campus, Izmit-Kocaeli, Turkey.

\author{
Communicated by E. Savaş
}

\begin{abstract}
Making use of the convolution operator we introduce a new class of analytic functions in the open unit disk and investigate some subordination results. (C)2017 All rights reserved.

Keywords: Analytic functions, univalent function, differential subordination, convex function, Hadamard product, best dominant.

2010 MSC: 30C45.
\end{abstract}

\section{Introduction}

Let $\mathbb{C}$ be complex plane and $\mathbb{U}=\{z \in \mathbb{C}:|z|<1\}=\mathbb{U} \backslash\{0\}$, open unit disc in $\mathbb{C}$. Let $H(\mathbb{U})$ be the class of analytic functions in $\mathbb{U}$. For $p \in N^{+}=\{1,2,3, \cdots\}$ and $a \in \mathbb{C}$, let $H[a, k]$ be the subclass of $H(\mathbb{U})$ consisting of the functions of the form

$$
f(z)=a+a_{k} z^{k}+a_{k+1} z^{k+1}+\cdots
$$

with $H_{0} \equiv H[0,1]$ and $H \equiv H[1,1]$. Let $A_{p}$ be the class of all analytic functions of the form

$$
f(z)=z^{p}+\sum_{k=p+1}^{\infty} a_{k} z^{k}
$$

in the open unit disk $\mathbb{U}$ with $A_{1}=A$. For functions $f \in A_{p}$ given by equation (1.1) and $g \in A_{p}$ defined by

$$
g(z)=z^{p}+\sum_{k=p+1}^{\infty} b_{k} z^{k},
$$

their Hadamard product (or convolution) [7] of $f$ and $g$ is defined by

$$
(f * g)(z):=z^{p}+\sum_{k=p+1}^{\infty} a_{k} b_{k} z^{k} .
$$

A function $f \in H(\mathbb{U})$ is univalent if it is one to one in $\mathbb{U}$. Let $S$ denote the subclass of $A$ consisting of

Email address: akgul@kocaeli.edu.tr (Arzu Akgül)

doi:10.22436/jnsa.010.03.08

Received 2016-08-13 
functions univalent in $\mathbb{U}$. If a function $f \in A$ maps $\mathbb{U}$ onto a convex domain and $f$ is univalent, then $f$ is called a convex function. Let

$$
K=\left\{f \in A: \mathfrak{R}\left\{1+\frac{z f^{\prime \prime}(z)}{f^{\prime}(z)}\right\}>0, z \in \mathbb{U}\right\}
$$

denote the class of all convex functions defined in $\mathbb{U}$ and normalized by $f(0)=0, f^{\prime}(0)=1$. Let $f$ and $F$ be members of $\mathrm{H}(\mathbb{U})$. The function $f$ is said to be subordinate to $\varphi$, if there exists a Schwarz function $w$ analytic in $\mathbb{U}$ with

$$
w(0)=0 \text { and }|w(z)|<1, \quad(z \in \mathbb{U}),
$$

such that

$$
f(z)=\varphi(w(z))
$$

We denote this subordination by

$$
f(z) \prec \varphi(z) \text { or } f \prec \varphi .
$$

Furthermore, if the function $\varphi$ is univalent in $\mathbb{U}$, then we have the following equivalence $[5,13]$

$$
\mathrm{f}(z) \prec \varphi(z) \Longleftrightarrow \mathrm{f}(0)=\varphi(0) \text { and } \mathrm{f}(\mathrm{U}) \subset \varphi(\mathrm{U}) .
$$

The method of differential subordinations (also known as the admissible functions method) was first introduced by Miller and Mocanu in 1978 [11] and the theory started to develop in 1981 [12]. All the details were captured in a book by Miller and Mocanu in 2000 [13]. Let $\Psi: \mathbb{C}^{3} \times \mathbb{U} \longrightarrow \mathbb{C}$ and $h$ be univalent in $\mathbb{U}$. If $p$ is analytic in $\mathbb{U}$ and satisfies the second-order differential subordination

$$
\Psi\left(\mathrm{p}(z), z \mathrm{p}^{\prime}(z), z \mathrm{p}^{\prime \prime}(z) ; z\right) \prec \mathrm{h}(z),
$$

then $p$ is called a solution of the differential subordination. The univalent function $q$ is called a dominant of the solution of the differential subordination or more simply dominant, if $p \prec q$ for all $p$ satisfying (1.2). A dominant $\mathrm{q}_{1}$ satisfying $\mathrm{q}_{1} \prec \mathrm{q}$ for all dominants (1.2) is said to be the best dominant of (1.2).

For functions $f, g \in A_{p}$, the linear operator $Q_{\lambda, p}^{m}: A_{p} \longrightarrow A_{p}(\lambda \geqslant 0, m \in N \cup\{0\})$ is defined by:

$$
\begin{aligned}
\mathrm{Q}_{\lambda, p}^{0}(f * g)(z) & =(f * g)(z), \\
Q_{\lambda, p}^{1}(f * g)(z) & =Q_{\lambda, p}((f * g)(z)) \\
& =(1-\lambda)(f * g)(z)+\frac{\lambda z}{p}((f * g)(z))^{\prime} \\
& =z^{p}+\sum_{k=p+1}^{\infty} \frac{p+\lambda(k-p)}{p} a_{k} b_{k} z^{k}, \\
Q_{\lambda, p}^{2}(f * g)(z) & =Q_{\lambda, p}\left[Q_{\lambda}, p(f * g)(z)\right] .
\end{aligned}
$$

Thus, we get

$$
\mathrm{Q}_{\lambda, p}^{\mathrm{m}}(\mathrm{f} * \mathrm{~g})(z)=\mathrm{Q}_{\lambda, \mathrm{p}}\left(\mathrm{Q}_{\lambda, \mathrm{p}}^{\mathrm{m}-1}(\mathrm{f} * \mathrm{~g})(z)\right)=z^{\mathrm{p}}+\sum_{\mathrm{k}=\mathrm{p}+1}^{\infty}\left(\frac{\mathrm{p}+\lambda(\mathrm{k}-\mathrm{p})}{\mathrm{p}}\right)^{\mathrm{m}} \mathrm{a}_{\mathrm{k}} \mathrm{b}_{\mathrm{k}} z^{\mathrm{k}}, \quad(\lambda \geqslant 0) .
$$

From (1.3) it can be easily seen that

$$
\frac{\lambda z}{p}\left(Q_{\lambda, p}^{m}(f * g)(z)\right)^{\prime}=Q_{\lambda, p}^{m+1}(f * g)(z)-(1-\lambda) Q_{\lambda, p}^{m}(f * g)(z), \quad(\lambda \geqslant 0) .
$$

The operator $Q_{\lambda, p}^{m}(f * g)$ was introduced and studied by Selveraj and Selvakumaran [19], Aouf and Mostafa [4], and for $p=1$ was introduced by Aouf and Mostafa [3]. Recent years, Özkan [16], Özkan 
and Altntaş [17], Lupaş [9], and Lupaş [10] (also see [1, 2]) investigated some applications and results of subordinations of analytic functions given by convolution. Also Bulut [6] used the same techniques by using Komatu integral operator. In some of this study, the results given by Lupaş [10] and Lupaş [9] were generalized. In order to prove our main results we need the following lemmas.

Lemma 1.1 ([8]). Let $\mathrm{h}$ be convex function with $\mathrm{h}(0)=\mathrm{a}$ and let $\gamma \in \mathbb{C}^{*}:=\mathbb{C} \backslash\{0\}$ be a complex number with $\mathfrak{R}\{\gamma\} \geqslant 0$. If $\mathrm{p} \in \mathrm{H}[\mathrm{a}, \mathrm{k}]$ and

$$
p(z)+\frac{1}{\gamma} z p^{\prime}(z) \prec h(z)
$$

then

$$
\mathrm{p}(z) \prec \mathrm{q}(z) \prec \mathrm{h}(z),
$$

where

$$
\mathrm{q}(z)=\frac{\gamma}{\mathrm{n} z^{\gamma / \mathrm{n}}} \int_{0}^{z} \mathrm{t}^{(\gamma / \mathrm{n})-1} \mathrm{~h}(\mathrm{t}) \mathrm{dt}
$$

The function $\mathrm{q}$ is convex and is the best dominant of the subordination (1.4).

Lemma 1.2 ([15]). Let $\mathfrak{R}\{\mu\}>0, n \in \mathbb{N}$, and let

$$
w=\frac{n^{2}+|\mu|^{2}-\left|n^{2}-\mu^{2}\right|}{4 n \mathfrak{n}\{\mu\}} .
$$

Let $\mathrm{h}$ be an analytic function in $\mathrm{U}$ with $\mathrm{h}(0)=1$ and suppose that

$$
\mathfrak{R}\left\{1+\frac{z \mathrm{~h}^{\prime \prime}(z)}{\mathrm{h}^{\prime}(z)}\right\}>-w .
$$

If

$$
p(z)=1+p_{n} z^{n}+p_{n+1} z^{n+1}+\cdots
$$

is analytic in $\mathbb{U}$ and

$$
p(z)+\frac{1}{\mu} z p^{\prime}(z) \prec h(z)
$$

then

$$
\mathrm{p}(z) \prec \mathrm{q}(z),
$$

where $\mathrm{q}$ is a solution of the differential equation

$$
\mathrm{q}(z)+\frac{\mathrm{n}}{\mu} z \mathrm{q}^{\prime}(z)=\mathrm{h}(z), \quad \mathrm{q}(0)=1,
$$

given by

$$
q(z)=\frac{\mu}{n z^{\sigma / n}} \int_{0}^{z} t^{(\mu / n)-1} h(t) d t \quad(z \in \mathbb{U}) .
$$

Moreover $\mathrm{q}$ is the best dominant of the subordination (1.5).

Lemma 1.3 ([14]). Let $r$ be a convex function in $\mathbb{U}$ and let

$$
h(z)=r(z)+n \beta z r^{\prime}(z), \quad(z \in \mathbb{U}),
$$

where $\beta>0$ and $n \in \mathbb{N}$. If

$$
p(z)=r(0)+p_{n} z^{n}+p_{n+1} z^{n+1}+\cdots, \quad(z \in \mathbb{U})
$$


is holomorphic in $\mathbb{U}$ and

$$
p(z)+\beta z p^{\prime}(z) \prec h(z)
$$

then

$$
p(z) \prec r(z),
$$

and this result is sharp.

In the present paper, making use of the subordination results of [13] and [18] we will prove our main results.

Definition 1.4. Let $\mathfrak{R}_{\lambda, m}(\beta)$ be the class of functions $f \in A$ satisfying

$$
\mathfrak{R}\left\{\left(Q_{\lambda}^{m}(f * g)(z)\right)^{\prime}\right\}>\beta,
$$

where $z \in \mathbb{U}, 0 \leqslant \beta<1$.

\section{Main results}

Theorem 2.1. The set $\Re_{\lambda, m}(\beta)$ is convex.

Proof. Let

$$
\left(\mathrm{f}_{\mathrm{j}} * \mathrm{~g}_{\mathrm{j}}\right)(z)=z+\sum_{\mathrm{k}=2}^{\infty} \mathrm{a}_{\mathrm{k}, \mathrm{j}} \mathrm{b}_{\mathrm{k}, \mathrm{j}} z^{\mathrm{k}} \quad(z \in \mathbb{U} ; \mathrm{j}=1, \ldots \mathrm{m})
$$

be in the class of $\mathfrak{R}_{\lambda, m}(\beta)$. Then, by Definition 1.4 , we get

$$
\mathfrak{R}\left\{\left(Q_{\lambda}^{m}\left(f_{j} * g_{j}\right)(z)\right)^{\prime}\right\}=\mathfrak{R}\left\{1+\sum_{k=2}^{\infty} a_{k, j} b_{k, j} k z^{k-1}\right\}>\beta .
$$

For any positive numbers $\lambda_{1}, \lambda_{2}, \ldots, \lambda_{\ell}$ such that

$$
\sum_{j=1}^{\ell} \lambda_{j}=1
$$

we have to show that the function

$$
h(z)=\sum_{j=1}^{\ell} \lambda\left(f_{j} * g_{j}\right)(z)
$$

is member of $\Re_{\lambda, m}(\beta)$, that is,

$$
\mathfrak{R}\left\{\left(Q_{\lambda}^{m} h(z)\right)^{\prime}\right\}>\beta .
$$

Thus, we have

$$
\mathrm{Q}_{\lambda}^{\mathrm{m}} \mathrm{h}(z)=z+\sum_{\mathrm{k}=2}^{\infty}(1+\lambda(k-1))^{\mathrm{m}}\left(\sum_{j=1}^{\ell} \lambda_{j} \mathrm{a}_{\mathrm{k}, \mathrm{j}} \mathrm{b}_{\mathrm{k}, \mathrm{j}}\right) z^{\mathrm{k}} .
$$

If we differentiate (2.2) with respect to $z$, then we obtain

$$
\left(Q_{\lambda}^{m} h(z)\right)^{\prime}=1+\sum_{k=2}^{\infty}(1+\lambda(k-1))^{m}\left(\sum_{j=1}^{\ell} \lambda_{j} a_{k, j} b_{k, j}\right) k z^{k-1}
$$




$$
=1+\sum_{j=1}^{\ell} \lambda_{j} \sum_{k=2}^{\infty}(1+\lambda(k-1))^{m} a_{k, j} b_{k, j} k z^{k-1} .
$$

Thus, we have

$$
\begin{aligned}
\mathfrak{R}\left\{\left(Q_{\lambda}^{m} h(z)\right)^{\prime}\right\} & =1+\sum_{j=1}^{\ell} \lambda_{j} \Re\left\{\sum_{k=2}^{\infty}(1+\lambda(k-1))^{m} a_{k, j} b_{k, j} k z^{k-1}\right\} \\
& >1+\sum_{j=1}^{\ell} \lambda_{j}(\beta-1) \\
& =\beta .
\end{aligned}
$$

Thus, the inequality (2.1) holds and we obtain desired result.

Theorem 2.2. Let $\mathrm{q}$ be convex function in $\mathbb{U}$ with $\mathrm{q}(0)=1$ and let

$$
h(z)=q(z)+\frac{1}{\gamma+1} z q^{\prime}(z) \quad(z \in \mathbb{U}),
$$

where $\gamma$ is a complex number with $\mathfrak{R}\{\gamma\}>-1$. If $\mathrm{f} \in \mathfrak{R}_{\sigma, \theta}(\beta)$ and $\mathfrak{F}=\Upsilon_{\gamma}(\mathrm{f} * \mathrm{~g})$, where

$$
\mathfrak{F}(z)=\Upsilon_{\gamma}(f * g)(z)=\frac{\gamma+1}{z^{\gamma}} \int_{0}^{z} t^{\gamma-1}(f * g)(t) d t,
$$

then,

$$
\left(Q_{\lambda}^{m}(f * g)(z)\right)^{\prime} \prec h(z)
$$

implies

$$
\left(Q_{\lambda}^{m} \mathfrak{F}(z)\right)^{\prime} \prec \mathrm{q}(z),
$$

and this result is sharp.

Proof. From the equality (2.3) we can write

$$
z^{\gamma} \mathfrak{F}(z)=(\gamma+1) \int_{0}^{z} t^{\gamma-1}(f * g)(t) d t,
$$

by differentiating the equality (2.5) with respect to $z$, we obtain

$$
(\gamma) \mathfrak{F}(z)+z \mathfrak{F}^{\prime}(z)=(\gamma+1)(f * g)(z) .
$$

If we apply the operator $Q_{\lambda}^{m}$ to the last equation, then we get

$$
(\gamma) Q_{\lambda}^{m} \mathfrak{F}(z)+z\left(Q_{\lambda}^{m} \mathfrak{F}(z)\right)^{\prime}=(\gamma+1) Q_{\lambda}^{m}(f * g)(z) .
$$

If we differentiate (2.6) with respect to $z$, we can obtain

$$
\left(Q_{\lambda}^{\mathrm{m}} \mathfrak{F}(z)\right)^{\prime}+\frac{1}{\gamma+1} z\left(Q_{\lambda}^{\mathrm{m}} \mathfrak{F}(z)\right)^{\prime \prime}=\left(\mathrm{Q}_{\lambda}^{\mathrm{m}} \mathrm{f}(z)\right)^{\prime} .
$$

By using the differential subordination given by (2.4) in the equality (2.7), we have

$$
\left(\mathrm{Q}_{\lambda}^{\mathrm{m}} \mathfrak{F}(z)\right)^{\prime}+\frac{1}{\gamma+1} z\left(\mathrm{Q}_{\lambda}^{\mathrm{m}} \mathfrak{F}(z)\right)^{\prime \prime} \prec \mathrm{h}(z)
$$


Now, we define

$$
p(z)=\left(Q_{\lambda}^{m} \mathfrak{F}(z)\right)^{\prime}
$$

Then by a simple computation we get

$$
p(z)=\left[z+\sum_{k=2}^{\infty}(1+\lambda(k-1))^{m} \frac{\gamma+1}{\gamma+k} a_{k} b_{k} z^{k}\right]^{\prime}=1+p_{1} z+p_{2} z+\cdots, \quad(p \in H[1,1]) .
$$

Using the equation (2.9) in the subordination (2.8), we obtain

$$
p(z)+\frac{1}{\gamma+1} z p^{\prime}(z) \prec h(z)=q(z)+\frac{1}{\gamma+1} z q^{\prime}(z) .
$$

If we use Lemma 1.2, then we get

$$
\mathrm{p}(z) \prec \mathrm{q}(z) .
$$

So we obtain the desired result and $q$ is the best dominant.

Example 2.3. If we choose in Theorem 2.2

$$
\gamma=i+1, \quad q(z)=\frac{1}{1-z},
$$

thus we get

$$
h(z)=\frac{(i+2)-(i+1) z}{(i+2)(1-z)^{2}} .
$$

If $(f * g) \in \mathfrak{R}_{\lambda, m}(\beta)$ and $\mathfrak{F}$ is given by

$$
\mathfrak{F}(z)=\Upsilon_{i}(f * g)(z)=\frac{i+2}{z^{i+1}} \int_{0}^{z} t^{i}(f * g)(t) d t
$$

then by Theorem 2.2, we obtain

$$
\left.\left(Q_{\lambda}^{m} f(z)\right)^{\prime} \prec h(z)=\frac{(i+2)-(i+1) z}{(i+2)(1-z)^{2}} \Longrightarrow\left(Q_{\lambda}^{m} \mathfrak{F}(z)\right)^{\prime} \prec q(z)\right)=\frac{1}{1-z} .
$$

Theorem 2.4. Let $\mathfrak{R}\{\gamma\}>-1$ and let

$$
w=\frac{1+|\gamma+1|^{2}-\left|\gamma^{2}+2 \gamma\right|}{4 \Re\{\gamma+1\}}
$$

Let $\mathrm{h}$ be an analytic function in $\mathbb{U}$ with $\mathrm{h}(0)=1$ and assume that

$$
\mathfrak{R}\left\{1+\frac{z \mathrm{~h}^{\prime \prime}(z)}{\mathrm{h}^{\prime}(z)}\right\}>-w .
$$

If $f * g \in \mathfrak{R}_{\lambda, \mathrm{m}}(\beta)$ and $\mathfrak{F}=\Upsilon_{\gamma}^{\delta}(\mathrm{f} * \mathrm{~g})$, where $\mathfrak{F}$ is defined by equation (2.3), then

$$
\left(Q_{\lambda}^{m}(f * g)(z)\right)^{\prime} \prec h(z)
$$

implies

$$
\left(Q_{\lambda}^{m} \mathfrak{F}(z)\right)^{\prime} \prec q(z),
$$


where $\mathrm{q}$ is the solution of the differential equation

$$
h(z)=\mathrm{q}(z)+\frac{1}{\gamma+1} z \mathrm{q}^{\prime}(z), \quad \mathrm{q}(0)=1,
$$

given by

$$
q(z)=\frac{\gamma+1}{z^{\gamma+1}} \int_{0}^{z} t^{\gamma}(f * g)(t) d t .
$$

Moreover $\mathrm{q}$ is the best dominant of the subordination (2.10).

Proof. If we choose $n=1$ and $\mu=\gamma+1$ in Lemma 1.2, then the proof is obtained by means of the proof of Theorem 2.4.

Letting

$$
h(z)=\frac{1+(2 \beta-1) z}{1+z}, \quad 0 \leqslant \beta<1
$$

in Theorem 2.4, we obtain the following result.

Corollary 2.5. If $0 \leqslant \beta<1,0 \leqslant \xi<1, \lambda \geqslant 0, \mathfrak{R}\{\gamma\}>-1$, and $\mathfrak{F}=\Upsilon_{\gamma}(\mathrm{f} * \mathrm{~g})$ is defined by the equation (2.3), then

$$
\Upsilon_{\gamma}\left(\Re_{\lambda, m}(\beta)\right) \subset \Re_{\lambda, m}(\xi),
$$

where

$$
\xi=\min _{|z|=1} \mathfrak{R}\{\mathbf{q}(z)\}=\xi(\gamma, \beta)
$$

and this result is sharp. Also,

$$
\xi=\xi(\gamma, \beta)=(2 \beta-1)+2(\gamma+1)(1-\beta) \tau(\gamma),
$$

where

$$
\tau(\gamma)=\int_{0}^{1} \frac{t^{\gamma}}{1+t} d t
$$

Proof. Let $f \in \mathfrak{R}_{\lambda, m}(\beta)$. Then from Definition 1.4 it is known that

$$
\mathfrak{R}\left\{\left(Q_{\lambda}^{m}(f * g)(z)\right)^{\prime}\right\}>\beta,
$$

which is equivalent to

$$
\left(Q_{\lambda}^{m}(f * g)(z)\right)^{\prime} \prec h(z) .
$$

By using Theorem 2.2, we have

$$
\left(\mathrm{Q}_{\lambda}^{\mathrm{m}} \mathfrak{F}(z)\right)^{\prime} \prec \mathrm{q}(z)
$$

If we take

$$
h(z)=\frac{1+(2 \beta-1) z}{1+z}, \quad 0 \leqslant \beta<1,
$$

then $h$ is convex and by Theorem 2.4, we obtain

$$
\left(Q_{\lambda}^{m} \mathfrak{F}(z)\right)^{\prime} \prec \mathrm{q}(z)=\frac{\gamma+1}{z^{\gamma+1}} \int_{0}^{z} \mathrm{t}^{\gamma} \frac{1+(2 \beta-1) \mathrm{t}}{1+\mathrm{t}} \mathrm{dt}=(2 \beta-1)+2 \frac{(1-\beta)(\gamma+1)}{z^{\gamma+1}} \int_{0}^{z} \frac{\mathrm{t}^{\gamma}}{1+\mathrm{t}} \mathrm{dt} .
$$

On the other hand if $\mathfrak{R}\{\gamma\}>-1$, then from the convexity of $q$ and the fact that $q(\mathbb{U})$ is symmetric with respect to the real axis, we get

$$
\mathfrak{R}\left\{\left(Q_{\lambda}^{m} \mathfrak{F}(z)\right)^{\prime}\right\} \geqslant \min _{|z|=1} \mathfrak{R}\{\mathbf{q}(z)\}=\mathfrak{R}\{\mathbf{q}(1)\}=\xi(\gamma, \beta)=2 \beta-1+2(1-\beta)(\gamma+1) \tau(\gamma),
$$


where $\tau(\gamma)$ is given by equation (2.12). From inequality (2.13), we get

$$
\Upsilon_{\gamma}\left(\Re_{\lambda, m}(\beta)\right) \subset \mathfrak{R}_{\lambda, m}(\xi),
$$

where $\xi$ is given by (2.11).

Theorem 2.6. Let $\mathrm{q}$ be a convex function with $\mathrm{q}(0)=1$ and $\mathrm{h}$ a function such that

$$
\mathrm{h}(z)=\mathrm{q}(z)+z \mathrm{q}^{\prime}(z), \quad(z \in \mathbb{U}) .
$$

If $\mathrm{f} \in \mathrm{A}$, then the following subordination

$$
\left(Q_{\lambda}^{m}(f * g)(z)\right)^{\prime} \prec h(z)=q(z)+z q^{\prime}(z)
$$

implies that

$$
\frac{\left(\mathrm{Q}_{\lambda}^{\mathrm{m}}(\mathrm{f} * \mathrm{~g})(z)\right)}{z} \prec \mathrm{q}(z),
$$

and the result is sharp.

Proof. Let

$$
p(z)=\frac{\left(Q_{\lambda}^{m}(f * g)(z)\right)}{z}
$$

Differentiating (2.15), we have

$$
\left(Q_{\lambda}^{\mathfrak{m}}(f * g)(z)\right)^{\prime}=p(z)+z p^{\prime}(z), \quad(z \in \mathbb{U})
$$

and thus (2.14) becomes

$$
\mathrm{p}(z)+z \mathrm{p}^{\prime}(z) \prec \mathrm{h}(z)=\mathrm{q}(z)+z \mathrm{q}^{\prime}(z) .
$$

Hence by applying Lemma1.3, we conclude that

$$
p(z) \prec q(z),
$$

that is,

$$
\frac{\left(\mathrm{Q}_{\lambda}^{\mathrm{m}}(\mathrm{f} * \mathrm{~g})(z)\right)}{z} \prec \mathrm{q}(z),
$$

and this result is sharp.

Theorem 2.7. Let $\mathrm{q}$ be a convex function with $\mathrm{q}(0)=1$ and $\mathrm{h}$ the function

$$
\mathrm{h}(z)=\mathrm{q}(z)+z \mathrm{q}^{\prime}(z) \quad(z \in \mathbb{U}) .
$$

If $\mathrm{m} \in \mathbb{N}, \mathrm{f} \in \mathrm{A}$ and verifies the differential subordination

$$
\left(\frac{Q_{\lambda}^{m+1}(f * g)(z)}{Q_{\lambda}^{m}(f * g)(z)}\right)^{\prime} \prec h(z),
$$

then

$$
\frac{\mathrm{Q}_{\lambda}^{\mathrm{m}+1}(\mathrm{f} * \mathrm{~g})(z)}{\mathrm{Q}_{\lambda}^{\mathrm{m}}(\mathrm{f} * \mathrm{~g})(z)} \prec \mathrm{q}(z),
$$

and this result is sharp.

Proof. For the function $f \in A$, given by the equation (1.1), we have

$$
\mathrm{Q}_{\lambda}^{\mathrm{m}}(\mathrm{f} * \mathrm{~g})(z)=z+\sum_{\mathrm{k}=2}^{\infty}(1+\lambda(k-1))^{\mathrm{m}} \frac{\gamma+1}{\mathrm{k}+\gamma} \mathrm{a}_{\mathrm{k}} \mathrm{b}_{\mathrm{k}} z^{\mathrm{k}}, \quad(z \in \mathbb{U}) .
$$


Let us consider

$$
\begin{aligned}
p(z)=\frac{Q_{\lambda}^{m+1}(f * g)(z)}{Q_{\lambda}^{m}(f * g)(z)} & =\frac{z+\sum_{k=2}^{\infty}(1+\lambda(k-1))^{m+1} \frac{\gamma+1}{k+\gamma} a_{k} b_{k} z^{k}}{z+\sum_{k=2}^{\infty}(1+\lambda(k-1))^{m} \frac{\gamma+1}{k+\gamma} a_{k} b_{k} z^{k}} \\
& =\frac{1+\sum_{k=2}^{\infty}(1+\lambda(k-1))^{m+1} \frac{\gamma+1}{k+\gamma} a_{k} b_{k} z^{k-1}}{1+\sum_{k=2}^{\infty}(1+\lambda(k-1))^{m} \frac{\gamma+1}{k+\gamma} a_{k} b_{k} z^{k-1}}
\end{aligned}
$$

We get

$$
(p(z))^{\prime}=\frac{\left(Q_{\lambda}^{m+1}(f * g)(z)\right)^{\prime}}{Q_{\lambda}^{m}(f * g)(z)}-p(z) \frac{\left(Q_{\lambda}^{m}(f * g)(z)\right)^{\prime}}{\left.Q_{\lambda}^{m}(f * g)(z)\right)}
$$

and

$$
\mathrm{p}(z)+z \mathrm{p}^{\prime}(z)=\left(\frac{z \mathrm{Q}_{\lambda}^{\mathrm{m}+1}(\mathrm{f} * \mathrm{~g})(z)}{\mathrm{Q}_{\lambda}^{\mathrm{m}}(\mathrm{f} * \mathrm{~g})(z)}\right)^{\prime} \quad(z \in \mathbb{U})
$$

Thus, the relation (2.16) becomes

$$
\mathrm{p}(z)+z \mathrm{p}^{\prime}(z) \prec \mathrm{h}(z)=\mathrm{q}(z)+z \mathrm{q}^{\prime}(z), \quad(z \in \mathbb{U}),
$$

and by using Lemma 1.3, we obtain

$$
\mathrm{p}(z) \prec \mathrm{q}(z)
$$

that is,

$$
\frac{\left(Q_{\lambda}^{m}(f * g)(z)\right)}{z} \prec q(z) .
$$

\section{Acknowledgment}

The author would like to thank the anonymous referees for their valuable suggestions which improved the presentation of the paper.

\section{References}

[1] Ş. Altınkaya, S. Yalçın, Coefficient estimates for two new subclasses of biunivalent functions with respect to symmetric points, J. Funct. Spaces, 2015 (2015), 5 pages. 1

[2] Ş. Altınkaya, S. Yalçın, General properties of multivalent concave functions involving linear operator of carlson-shaffer type, Comptes rendus de 1Acade'mie bulgare des Sciences, 69 (2016), 1533-1540. 1

[3] M. K. Aouf, A. O. Mostafa, Sandwich theorems for analytic functions defined by convolution, Acta Univ. Apulensis Math. Inform., 21 (2010), 7-20. 1

[4] M. K. Aouf, A. O. Mostafa, Subordination results for a class of multivalent non-Bazilevic analytic functions defined by linear operator, Acta Univ. Apulensis Math. Inform., 31 (2012), 307-320. 1

[5] T. Bulboacă, Differential subordinations and superordinations, Recent Results, Casa Cărţii de Ştiinţă, Cluj-Napoca, (2005). 1

[6] S. Bulut, Some applications of second-order differential subordination on a class of analytic functions defined by Komatu integral operator, ISRN Math. Anal., 2014 (2014), 5 pages. 1

[7] P. L. Duren, Univalent functions, Grundlehren der Mathematischen Wissenschaften [Fundamental Principles of Mathematical Sciences], Springer-Verlag, New York, (1983) 1

[8] D. J. Hallenbeck, S. Ruscheweyh, Subordination by convex functions, Proc. Amer. Math. Soc., 52 (1975), 191-195. 1.1 
[9] A. A. Lupaş, A new comprehensive class of analytic functions defined by multiplier transformation, Math. Comput. Modelling, 54 (2011), 2355-2362. 1

[10] A. A. Lupas,, Differential subordinations using multiplier transformation, Adv. Appl. Math. Sci., 14 (2015), 71-89. 1

[11] S. S. Miller, P. T. Mocanu, Second-order differential inequalities in the complex plane, J. Math. Anal. Appl., 65 (1978), 298-305. 1

[12] S. S. Miller, P. T. Mocanu, Differential subordinations and univalent functions, Michigan Math. J., 28 (1981), $157-171$. 1

[13] S. S. Miller, P. T. Mocanu, Differential subordinations, Theory and applications, Monographs and Textbooks in Pure and Applied Mathematics, Marcel Dekker, Inc., New York, (2000). 1, 1

[14] P. T. Mocanu, T. Bulboacă, G. Sălăgean, Geometric theory of univalent functions, Casa Cărţii de Ştiinţă, Cluj-Napoca, (1999). 1.3

[15] G. Oros, G. I. Oros, A class of holomorphic functions II, Miron Nicolescu (19031975) and Nicolae Ciorănescu (19031957), Libertas Math., 23, (2003), 65-68. 1.2

[16] Ö. Özkan, Some subordination results of multivalent functions defined by integral operator, J. Inequal. Appl., 2007 (2007), 8 pages. 1

[17] Ö. Özkan, O. Altntaş, Applications of differential subordination, Appl. Math. Lett., 19 (2006), 728-734. 1

[18] G. S. Sălăgean, Subclasses of univalent functions, Complex analysis-fifth Romanian-Finnish seminar, Part 1, Bucharest, (1981), 362-372, Lecture Notes in Math., Springer, Berlin, 1013 (1983). 1

[19] C. Selvaraj, K. A. Selvakumaran, On certain classes of multivalent functions involving a generalized differential operator, Bull. Korean Math. Soc., 46 (2009), 905-915. 1 